\title{
Die Implementation einer Intervention zur Veränderung des Selbstkonzepts im Sportunterricht
}

\section{Esther Oswald, Stefan Valkanover und Achim Conzelmann}

Bei Studien zur Selbstkonzeptentwicklung fehlt meist der Hinweis auf die Programmumsetzung oder Implementation. Diesem Defizit der Implementationskontrolle nimmt sich die vorliegende Studie an und befasst sich mit der Messung und Erklärung der Implementationshäufigkeit im Rahmen einer Intervention zu Selbstkonzept förderndem Sportunterricht $\left(N_{\text {Lehrer }}=16, N_{\text {Schüler }}=309\right)$. Die Ergebnisse zeigen, dass Lehrpersonen eine individuumsorientierte, reflexive Sportvermittlung im Sportunterricht einsetzen. Die Implementationshäufigkeit der Lehrpersonen hängt von deren Berufserfahrung ab. Eine häufigere Implementation geht bei Schülerinnen und Schülern mit einer stärkeren Veränderung des Körperselbstwerts, jedoch nicht des sportbezogenen Fähigkeitsselbstkonzepts, einher.

Die Implementation einer Intervention zur Veränderung des Selbstkonzepts im Sportunterricht

\section{Einleitung}

Im letzten Jahrzehnt haben sich innerhalb der Unterrichtswissenschaft diverse Arbeitsgruppen der Untersuchung der Selbstkonzeptentwicklung angenommen (z. B. Aust, Watermann \& Grube, 2010; Filipp, 2006; Lüdtke \& Köller, 2002), so auch in der Sportwissenschaft (z. B. Stiller \& Alfermann, 2005). In zunehmendem Masse werden dabei quasi-experimentelle Interventionsstudien (z. B. Conzelmann, Schmidt \& Valkanover, 2011; Fox, 2000) durchgeführt, wobei Aspekte der Programmumsetzung oder Implementation allerdings selten ausgewiesen werden. Ohne Kenntnisse bezüglich Dosierung, Quantität und Qualität (Dane \& Schneider, 1998) der Implementation lassen sich Programmwirkungen, z. B. von Lehrpersonenfortbildungen, jedoch nicht umfassend erklären.

In der vorliegenden Studie wird im Rahmen einer Intervention zur Selbstkonzeptentwicklung im Sportunterricht eine Möglichkeit der Messung der Implementation präsentiert und (1) untersucht, von welchen Aspekten eine 
hohe Implementationshäufigkeit abhängt. Zudem wird (2) analysiert, ob eine hohe Implementationshäufigkeit auf Seiten der Lehrperson auch mit entsprechenden Effekten im Selbstkonzept der Schülerinnen und Schüler einhergeht.

\section{Begriffsbestimmung Implementation}

Unter Implementation wird die Umsetzung wissenschaftlicher Erkenntnisse in die gesellschaftliche Praxis verstanden (Euler \& Sloane, 1998, S. 312). Im Schulalltag bezieht sich diese auf die Wirksamkeit von Lehr- und Lernmaterialien (Lütgert \& Stephan, 1983, S. 502). Es geht um die Frage, «wie ein Konzept, eine Theorie ... in praktisches Handeln umgesetzt werden können» (Euler \& Sloane, 1998, S. 313). Erziehungswissenschaftlich relevant ist z. B. die Umsetzung didaktischer Theorien von Lehrpersonen im Unterricht. In Bezug auf didaktisches Handeln meint Implementation, dass Lehrpersonen bestimmte Vorgaben in ihren Unterricht einbeziehen und unter Berücksichtigung der jeweiligen situativen und personalen Möglichkeiten entsprechend handeln. Allerdings ist zu bedenken, dass Lehrpersonen (didaktische) Theorien erstens nicht technologisch auf Schülerinnen und Schüler anwenden (sog. Technologiedefizit der Pädagogik; u. a. Döring, 1989). Lehrpersonen handeln jeweils vor dem Hintergrund grosser Unterrichtskomplexität (Herzog, 2002, S. 433). Intentionales Handeln der Lehrperson ist damit nicht hinreichend für einen Lernerfolg auf Schülerinnenund Schülerseite. Zweitens reicht die alleinige Vermittlung von Wissen an Lehrpersonen nicht aus, um bei diesen überdauernde Verhaltensmodifikationen auszulösen (Radtke, 1996) resp. um eine umfassende Implementation vorgegebener didaktischer Theorien anzuleiten. Von Implementation kann deshalb im Unterricht bereits gesprochen werden, wenn Lehrpersonen Vorgaben «möglichst wie vorgesehen» realisieren (Gräsel \& Parchmann, 2004, S. 199).

Mit dem Ausmass der Implementation beschäftigt sich die Implementationsgenauigkeit. Sie gibt an, wie genau ein Programm entsprechend den Vorgaben umgesetzt wird und bezieht sich auf die Aspekte Umfang und Häufigkeit der Implementation, richtige Vermittlung der Interventionsinhalte, Qualität der Wiedergabe der Interventionsinhalte und Ausmass der Involvierung der Teilnehmenden in die Interventionsaktivität (Mihalic, 2004; Dane \& Schneider, 1998). Die Implementationskontrolle hat zum Ziel, (Interventions-)Massnahmen bei zukünftigem Einsatz zu optimieren und allfällige Wirkungen (interne Validität; Gollwitzer \& Jäger, 2007, S. 129) zu erklären.

\section{Implementation innerhalb der Unterrichtswissenschaft} Aufgrund der aktuellen Output-Orientierung des Bildungswesens und einer Häufung an Bildungsprogrammen und -reformen gewinnt die Implementationsforschung in der Unterrichtswissenschaft zunehmend an Bedeutung. Trotzdem besteht nach wie vor ein Mangel an Angaben zur Implementation von Programmen und an Implementationsforschung (Gräsel \& Parchmann, 2004, S. 197). Gräsel und Parchmann weisen darauf hin, dass es Aufgabe der 
unterrichtswissenschaftlichen Forschung ist, «Wissen darüber bereit zu stellen, wie ihre Ergebnisse verbreitet werden können, was geeignete und weniger geeignete Implementationsstrategien sind, ... woran Implementation scheitern kann» (a.a.O.). Böttcher (2002, S. 206) beschreibt, dass ein allgemeines Desinteresse vorliegt, «die Wirksamkeit neuer pädagogischer Programme empirisch zu analysieren und zu kontrollieren». Grund dafür ist unter anderem die Schwierigkeit, operationalisierbare Kriterien für komplexe Konstrukte wie «gutes Lehrerhandeln" oder "personale Kompetenzen» zu finden (Brohm, 2006, S. 248-250), was eine umfassende Messung und Analyse der Implementation und das Erbringen von Nachweisen bei Interventionsstudien im schulischen Setting beeinträchtigt. Die Implementation wird folglich nur in wenigen pädagogischen (Selbstkonzept-)Interventionsstudien (z. B. Drössler, Jerusalem \& Mittag, 2007) miterhoben und publiziert, wobei dort von grösseren Programmwirkungen bei grösserer Implementationshäufigkeit berichtet wird.

Da Programme und Interventionen in der Regel darauf abzielen, (Lern-) Prozesse und Fertigkeiten der Schülerinnen und Schüler zu verbessern, sollte die Implementationskontrolle nicht (nur) aus einer Evaluation der Selbsteinschätzung der Lehrpersonen bestehen (Zedler, Fischler, Kirchner \& Schröder, 2004, S. 116), sondern auch Effekte bezüglich der Lernprozesse bei Schülerinnen und Schülern berücksichtigen (Gräsel et al., 2004, S. 134).

\section{Determinanten der Implementation}

Bezüglich einer erfolgreichen Implementation spielen personale Merkmale der Umsetzenden und flankierende Massnahmen einer Intervention eine entscheidende Rolle (Gräsel \& Parchmann, 2004, S. 201). Allerdings besteht bezüglich konkreter hemmender und fördernder Faktoren einer Implementation ein Forschungsdefizit (Gräsel \& Parchmann, 2004, S. 197).

Zum Einfluss personaler Merkmale der Lehrperson auf die Implementation sind uns kaum Studien bekannt. Subjektive Theorien der Lehrperson spielen bei der Übernahme von schulischen Neuerungen eine zentrale Rolle (Gräsel \& Parchmann, 2004, S. 201; Lütgert \& Stephan, 1983, S. 516). Inwiefern eine Lehrperson veränderte unterrichtliche Vorgaben übernimmt, hängt davon $a b$, wie realisierbar, nützlich oder sinnvoll Massnahmen empfunden werden (Gräsel \& Parchmann, 2004, S. 203). Ebenfalls wichtig sind Selbstwirksamkeitserwartungen (Bandura, 1997). Schmitz und Schwarzer (2002, S. 208) zeigen, dass eine hohe Selbstwirksamkeit mit einem erhöhten pädagogischen Engagement und geringerer Überlastung einhergehen. Entscheidende Moderatoren der Implementation könnten zudem das Alter und die Berufserfahrung der Lehrperson sein.

Bezüglich flankierender Massnahmen zur Erreichung einer möglichst optimalen Implementation sind insbesondere drei Aspekte hervorzuheben (Gräsel \& Parchmann, 2004; Paul \& Volk, 2002; Reinmann-Rothmeier \& Mandl, 1998): Erstens ist die Nähe der Interventionsinhalte zur untersuchten Praxis entscheidend. Zweitens spielt der Einbezug von Lehrerkollegien eine 
wichtige Rolle, weil sich Lehrpersonen bei der Implementation gegenseitig unterstützen können. Drittens ist die langfristige Unterstützung der Lehrpersonen entscheidend.

\section{Gegenstand der Implementation: Selbstkonzeptverände- rungen im sportunterricht}

Eine zentrale Rolle bei der Genese des Selbstkonzepts (Shavelson, Hubner \& Stanton, 1976) spielen die verschiedenen Prädikaten(selbst-)zuweisungen (Filipp, 1979). Deren Hauptquellen sind im Kindesalter, nebst dem Nachdenken über das eigene Tun, verbale und nonverbale Rückmeldungen von Gleichaltrigen und Lehrpersonen. Diese in Form von Wissen über die eigene Person gespeicherten Informationen stellen das Selbstkonzept dar. Im Sportunterricht steht die Veränderung des physischen Selbstkonzepts im Zentrum. Dies geschieht in der Regel bottom-up aufgrund von Veränderungen im sportbezogenen Fähigkeitsselbstkonzept (Brettschneider \& Gerlach, 2004) und im Körperselbstwert (Sonstroem \& Morgan, 1989; Stiller \& Alfermann, 2008). Das sportbezogene Fähigkeitsselbstkonzept umfasst sämtliche Selbstbewertungen der eigenen sportlichen Leistungsfähigkeit, während unter Körperselbstwert als evaluative Komponente des physischen Selbstkonzepts die Zufriedenheit mit dem eigenen Körper verstanden wird. Die entscheidende Rolle spielen durch sportliche Aktivitäten ermöglichte bereichsspezifische physische Selbstwirksamkeits- und Kompetenzerfahrungen, welche wiederum das sportbezogene Fähigkeitsselbstkonzept und den Körperselbstwert beeinflussen.

Damit sportliche Aktivitäten Einfluss auf die oben erwähnten Konzepte haben, was in Lehrplänen als Richtziel für Sportunterricht genannt wird (z. B. Erziehungsdirektion Kanton Bern, 2004), müssen diese Aktivitäten Selbstkonzept fördernd gestaltet werden. In Anlehnung an die verschiedenen Quellen der Selbstkonzeptgenese (Prädikatenzuweisungen, Filipp, 1979) basiert Selbstkonzept fördernder Sportunterricht insbesondere auf der Umsetzung dreier didaktisch-methodischer Prinzipien durch die Lehrperson (Conzelmann et al., 2011): Das Prinzip der Kompetenzerfahrung (1) umfasst die Förderung der sportlichen Kompetenzen von Schülerinnen und Schülern mittels angemessener Aufgabenschwierigkeiten und präziser Rückmeldungen durch die Lehrperson, was bei Schülerinnen und Schülern entsprechende Selbstwirksamkeitserfahrungen ermöglicht. Das Prinzip der reflexiven Sportvermittlung (2) beinhaltet Unterbrechungen des Unterrichts (Time-out) durch die Lehrperson, in welchen die Schülerinnen und Schüler dazu angeleitet werden, über ihre Erfahrungen bezüglich der eigenen Fertigkeiten und Einstellungen nachzudenken. Diese Selbstbeobachtung mittels inneren Dialogs ist für die Ausdifferenzierung des Selbstkonzepts zentral (Filipp, 1979). Ausgangspunkt des Prinzips der individualisierten Lernbegleitung (3) ist die Leistungsheterogenität im Sportunterricht. Selbstkonzept fördernder Sportunterricht sollte deshalb unter Verwendung der individuellen Bezugsnorm (Rheinberg, 2008) bei Rückmeldungen und entspre- 
chender Unterrichtsgestaltung die unterschiedlichen Bedürfnisse der Schülerinnen und Schüler berücksichtigen. Im Rahmen von Selbstkonzept förderndem Sportunterricht kann unter Berücksichtigung dieser drei Prinzipien von einer individuumsorientierten, reflexiven Sportvermittlung gesprochen werden.

\section{Fragestellungen und Hypothesen}

Vor der hier dargestellten Intervention wurden Lehrpersonen in einem Workshop geschult, Selbstkonzept fördernden Sportunterricht mittels einer individuumsorientierten, reflexiven Sportvermittlung zu erteilen (vgl. Conzelmann et al., 2011). Nach einer vorausgehenden Analyse der Implementationshäufigkeit der Lehrpersonen wird in der vorliegenden Studie (1) untersucht, von welchen Aspekten eine hohe Implementationshäufigkeit abhängt:

Unterscheiden sich Lehrpersonen, die häufig eine individuumsorientierte, reflexive Sportvermittlung im Unterricht einsetzen, bezüglich ihrer Einstellungen zur Intervention, Selbstwirksamkeit, Alter und Berufserfahrung von denjenigen Lehrpersonen, die eine individuumsorientierte, reflexive Sportvermittlung weniger häufig einsetzen?

Mittels der folgenden, aufgrund der theoretischen Vorüberlegungen ableitbaren Hypothese wird (2) überprüft, wie sich eine unterschiedliche Implementationshäufigkeit einer individuumsorientierten, reflexiven Sportvermittlung auf die Veränderung des Selbstkonzepts der Schülerinnen und Schüler auswirkt:

Eine häufige individuumsorientierte, reflexive Sportvermittlung wirkt sich positiv auf das sportbezogene Fähigkeitsselbstkonzept und den Körperselbstwert von Schülerinnen und Schülern aus.

\section{Methodik}

\section{Design}

Die Studie, die innerhalb der Berner Interventionsstudie Schulsport (Conzelmann et al., 2011) zu verorten ist, ist als quasi-experimentelle Längsschnittstudie angelegt. Die Intervention bestand aus einer Treatmentphase von zehn Wochen. Innerhalb dieser zehn Wochen führte jede Lehrperson mit ihrer Klasse eines von drei spezifisch gestalteten Modulen (Leistung, Wagnis und Spiel) mittels individuumsorientierter, reflexiver Sportvermittlung durch. Die Intervention fand pro Woche jeweils in zwei der drei obligatorischen Sportunterrichtslektionen à je 45 Minuten statt.

\section{St ichprobe}

Die Stichprobe besteht aus Schweizer Sportlehrpersonen ( $N=16,63$ \% Lehrerinnen, Alter: $M=32.5)$ der fünften Klasse des Kantons Bern sowie ihren Primarschülern $(N=309,49 \%$ Schülerinnen, Alter: $M=11.9)$. Die Teilnehmenden gehören 16 verschiedenen Schulklassen an und wurden von den jewei- 
ligen Schulleitungen über die Möglichkeit der Studienteilnahme informiert. Sechs Klassen führten das Modul Leistung $\left(n_{\text {Lehrer }}=6, n_{\text {Schüler }}=114\right)$ und je fünf Klassen das Modul Wagnis $\left(n_{\text {Lehrer }}=5, n_{\text {Schüler }}=95\right)$ oder das Modul Spiel $\left(n_{\text {Lehrer }}=5, n_{\text {Schüler }}=100\right)$ durch. Aufgrund fehlender Werte weniger Schülerinnen und Schüler bei einzelnen Variablen weichen die in die einzelnen Berechnungen einbezogenen Stichprobengrössen maximal $4.9 \%$ von der Gesamtstichprobe ab.

\section{Untersuchungs instrumente}

Selbsteinschätzung der Lehrperson bezüglich der Implementationshäufigkeit einer individuumsorientierten, reflexiven Sportvermittlung. Bei den Lehrpersonen wurden im Rahmen der Implementationshäufigkeit einer individuumsorientierten, reflexiven Sportvermittlung nach dem Interventionszeitraum die Selbsteinschätzung der Einsatzhäufigkeit des Lernjournals sowie der umgesetzten Reflexionsphasen und die Feedbackhäufigkeit erhoben. Die Skala «Häufigkeit der Interventionen» wurde explizit für die Berner Interventionsstudie Schulsport (Conzelmann et al., 2011) erstellt und besteht aus drei Fragen. Eine Beispielfrage lautet "Wie häufig habt ihr das Lernjournal während der Intervention eingesetzt?». Die Fragen zu Reflexionsphasen und Feedback sind analog gestaltet. Die Zustimmung der Fragen zu Reflexionsphasen und Feedbackhäufigkeit erfolgt auf einer vierstufigen Skala von $1=$ «nie» bis $4=$ «sehr oft», bei der Frage der Einsatzhäufigkeit des Lernjournals kann eine zutreffende Ziffer angegeben werden. Zur Reliabilität der Skala lässt sich keine Angabe machen, da die verschiedenen Aspekte jeweils mit einer einzelnen Frage erhoben wurden. Bezüglich Objektivität ist zu beachten, dass es sich um eine Selbsteinschätzung der Lehrperson handelt und die Skala damit keine Auskunft über die tatsächliche Einsatzhäufigkeit des Lernjournals, der Reflexionsphasen oder des Feedbacks durch die Lehrperson gibt. Da die vierstufige Antwortskala zudem Interpretationsspielraum zulässt, ist auch die Validität der Skala eingeschränkt.

Einstellungen gegenüber der Intervention, Selbstwirksamkeit, Alter und Berufserfahrung. Bei den Lehrpersonen wurden zur Überprüfung der Determinanten der Implementationshäufigkeit deren Einstellungen gegenüber der Intervention nach dem Interventionszeitraum sowie die Selbstwirksamkeit, das Alter und die Berufserfahrung vor dem Interventionszeitraum erhoben. Die Einstellungen gegenüber der Intervention wurden anhand dreier Skalen (Lernjournal, Reflexionsphasen- und Feedbackeinsatz) erhoben, bei welchen die Lehrpersonen angeben, wie sinnvoll, wichtig und hilfreich sie die Intervention einschätzen. Diese drei Skalen wurden explizit für die Berner Interventionsstudie Schulsport (Conzelmann et al., 2011) erstellt. Eine Beispielfrage lautet «Für wie sinnvoll erachtet ihr den Einsatz des Lernjournales für die Intervention?». Die Zustimmung erfolgt jeweils auf einer vierstufigen Skala von $1=$ "gar nicht sinnvoll / wichtig / hilfreich" bis $4=$ "sehr sinnvoll / wichtig / hilfreich». Die sieben verwendeten Items ${ }^{1}$ zur Selbstwirksamkeit der Lehrpersonen stammen aus der Dokumen- 
tation der Skala Lehrer-Selbstwirksamkeit (WirkLehr) von Schwarzer und Schmitz (1999). Das Item «Ich traue mir zu, die Schüler für neue Projekte zum Modellversuch zu begeistern" wurde durch "Ich traue mir zu, die Schüler für unser Projekt zu begeistern» umformuliert. Die Zustimmung erfolgt auf einer vierstufigen Skala von $1=$ "trifft gar nicht $z u$ » bis $4=$ "trifft voll $z u »$. Die verwendete Skala weist eine interne Konsistenz von Cronbachs $\alpha_{(\mathrm{T} 2)}=.69$ auf.

Objektive Implementationshäufigkeit einer individuumsorientierten, reflexiven Sportvermittlung. Im Rahmen einer individuumsorientierten, reflexiven Sportvermittlung wurde von den Schülerinnen und Schülern ein modulspezifisches, im Rahmen reflexiver Time-outs regelmässig individuell bearbeitetes Lernjournal geführt (vgl. Abbildung 1). Das Lernjournal beinhaltete verschiedene, dem Modul angepasste Arbeitsaufträge mit vorwiegend geschlossenen Fragen zur Selbsteinschätzung bezüglich der eigenen körperlichen Leistungsfähigkeit und der Zufriedenheit mit dem eigenen Körper. Zur Überprüfung der Implementationshäufigkeit wurde das im Unterricht ausgefüllte Lernjournal nach dem Interventionszeitraum eingesammelt und die Anzahl der Einträge pro Schülerin und Schüler erhoben. Als ein Eintrag gelten alle Fragen, die inhaltlich übereinstimmend en bloc zu einem Zeitpunkt beantwortet werden.
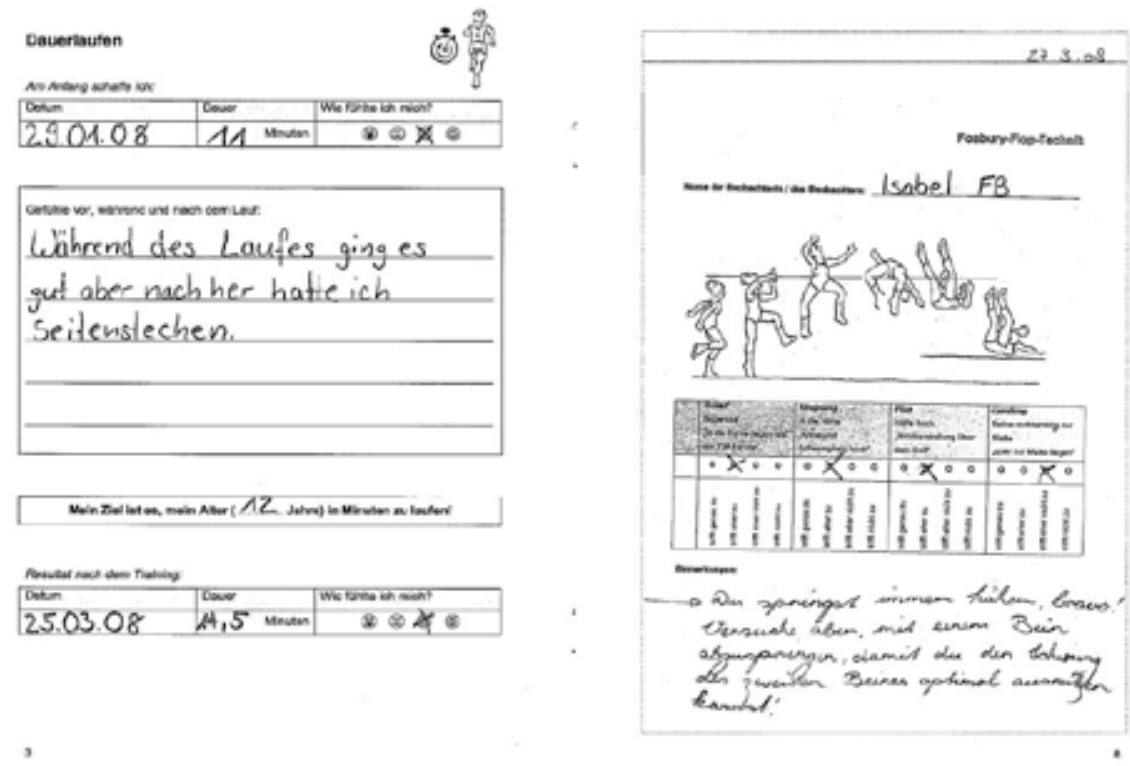

Abbildung 1: Auszug aus einem Lernjournal des Moduls «Leistung» mit einem Reflexionseintrag einer Schülerin und der Rückmeldung der Lehrperson

Sportbezogenes Fähigkeitsselbstkonzept und Körperselbstwert. Vor und nach dem Interventionszeitraum wurden das sportbezogene Fähigkeitsselbstkonzept und 
der Körperselbstwert der Schülerinnen und Schüler erhoben. Die Skala des sportbezogenen Fähigkeitsselbstkonzepts (Brettschneider \& Gerlach, 2004) stammt ursprünglich aus der Perceived Competence Scale for Children (Harter, 1985) und wurde bereits in der SPRINT-Studie (Deutscher Sportbund, 2006) eingesetzt. Sie besteht aus sechs Items, wobei die Zustimmung auf einer vierstufigen Skala von 1 = «stimmt nicht» bis $4=$ «stimmt genau» erfolgt. Ein Beispielitem lautet "Ich lerne sehr schnell neue Übungen beim Sport». Die verwendete Skala weist eine interne Konsistenz von Cronbachs $\alpha_{(\mathrm{T} 1 / \mathrm{T} 2)}=.80 / .83$ auf. Die Skala des Körperselbstwerts stammt aus der SPRINT-Studie (Deutscher Sportbund, 2006) und wurde in Anlehnung an das Physical Self-Perception Profile von Fox (1990) entwickelt. Sie besteht aus vier Items, welchen ebenfalls auf der vierstufigen Skala von $1=$ «stimmt nicht» bis $4=$ «stimmt genau» zugestimmt werden kann. Ein Beispielitem lautet "Ich bin mit meinem Körper sehr zufrieden". Die verwendete Skala weist eine interne Konsistenz von Cronbachs $\alpha_{(\mathrm{T} 1 / \mathrm{T} 2)}=.85 / .84$ auf. Die erhobenen internen Konsistenzen liegen damit im akzeptierbaren Bereich.

\section{Untersuchungsdurchführung}

Die Intervention bestand aus drei Modulen zu Lernzielen in den Bereichen Leistung, Wagnis und Spiel. Alle drei Module hatten eine positive Veränderung des sportbezogenen Fähigkeitsselbstkonzepts und des Körperselbstwerts zum Ziel. Die Zielsetzungen entsprachen den Vorgaben des Lehrplans (Erziehungsdirektion Kanton Bern, 2004) und die Inhalte richteten sich nach der Schweizer Lehrmittelreihe "Sporterziehung in der Schule» (Bucher, 1997). Das Modul Leistung beinhaltete Formen des Ausdauer- und Krafttrainings sowie des Hürdenlaufs und Hochsprungs, das Modul Wagnis Übungen im Geräte- und Bodenturnen sowie zusätzliche Mutposten und das Modul Spiel kleine Spiele, Kooperationsspiele und Spielentwicklungsreihen. Der Fokus der Intervention lag, nebst den spezifischen Inhalten, auf der Umsetzung einer individuumsorientierten, reflexiven Sportvermittlung. Diese bestand, nebst der entsprechenden Unterrichtsgestaltung und den angepassten Rückmeldungen der Lehrperson, aus einem modulspezifischen Lernjournal. Die Schülerinnen und Schüler formulierten dabei im Sportunterricht regelmässig individuelle Teilziele, hielten deren Überprüfung darin fest und reflektierten diese. Die Lehrpersonen gaben regelmässig schriftliche Rückmeldungen dazu ab. So konnten die Schülerinnen und Schüler ihre eigenen Lernfortschritte erkennen und entsprechende Selbstwirksamkeits- und Kompetenzerfahrungen wurden initiiert.

$\mathrm{Zu}$ jedem Modul wurde eine halbtägige Lehrpersonenschulung durchgeführt. Dabei wurden die theoretischen Grundlagen und die Inhalte der Intervention präsentiert sowie die individuumsorientierten, reflexiven Inszenierungsformen in der Sporthalle praktisch-methodisch bearbeitet. Zudem wurden folgende Massnahmen zur Sicherung einer möglichst optimalen Implementation (vgl. Kapitel 1.3) ergriffen: (1) Praxisnahe Gestaltung der Fortbildungsinhalte mit Orientierung am bestehenden Lehrmittel, (2) Vermittlung der Inhalte an für den Unterricht 
direkt verwendbaren Unterrichtsmaterialien, (3) Darstellung der Theorie anhand von Fallbeispielen, (4) Begleitung und Betreuung der Lehrpersonen durch die Studienleiter während der gesamten Intervention (Unterrichtsbesuche).

\section{Untersuchungsauswertung}

Die Untersuchungsauswertung erfolgte in drei Schritten. (1) In einer vorausgehenden deskriptiven Analyse der Implementationshäufigkeit wurden die ausgezählten Lernjournaleinträge der Schülerinnen und Schüler und die Selbsteinschätzung der Lehrpersonen bezüglich Einsatzhäufigkeit des Lernjournals, der Reflexionsphasen und des Feedbacks ausgewertet. (2) Zur Beantwortung der ersten Fragestellung wurden die Lehrpersonen anhand des Klassenmittelwerts der Anzahl Lernjournaleinträge ihrer Schülerinnen und Schüler (aufgrund der geringen Stichprobenzahl) in zwei Gruppen (niedrige vs. hohe Implementationshäufigkeit) eingeteilt (Modulweiser ${ }^{2}$ Mediansplit $^{3} ; n_{\text {niedrig }}=6, n_{\text {hoch }}=10$ ). Anschliessend wurde mittels zweiseitigen $t$-Tests (bei einer $\alpha$-Fehlerwahrscheinlichkeit von $5 \%$ ) geprüft, ob sich die Lehrpersonen aus den beiden Gruppen bezüglich Einstellungen zur Intervention, Selbstwirksamkeit, Alter und Berufserfahrung unterscheiden. (3) Zur Untersuchung der vorgehend formulierten Hypothese wurden die Schülerinnen und Schüler anhand der Anzahl Lernjournaleinträge in drei Gruppen eingeteilt (niedrige, mittlere und hohe Implementationshäufigkeit). Dazu wurden zuerst die Anzahl Lernjournaleinträge der Schülerinnen und Schüler modulweise z-standardisiert. Anschliessend wurden die Schülerinnen und Schüler modulübergreifend anhand ihres z-Werts in die drei Gruppen eingeteilt $\left(n_{\text {niedrig }}=48, n_{\text {mittel }}=197, n_{\text {hoch }}=49\right)$. Die Dreiteilung kommt durch Cuts bei +/-1 Standardabweichung zustande. Schliesslich wurde mittels zweifaktorieller Varianzanalyse mit Messwiederholung (bei einer $\alpha$-Fehlerwahrscheinlichkeit von $5 \%$ ) überprüft, ob sich die Veränderungen des sportbezogenen Fähigkeitsselbstkonzepts und des Körperselbstwerts der drei Implementationshäufigkeitsgruppen unterscheiden.

Die Anzahl Lernjournaleinträge werden zur Beantwortung der ersten Fragestellung und der Hypothese als Kriterium zur Gruppenbildung für die Implementationshäufigkeit verwendet. Diese Entscheidung beruht auf der Objektivität dieses Instruments und der damit implizierten tatsächlichen Schülerinnen- und Schülerbetätigung. Die Anzahl Lernjournaleinträge entspricht modulweise in etwa den Selbsteinschätzungen der Lehrpersonen zur verwendeten Häufigkeit des Lernjournals, der Reflexion und des Feedbacks ${ }^{4}$.

\section{Ergebnisse}

Im Folgenden wird die Implementationshäufigkeit der Lehrpersonen dargestellt, bevor auf die Ergebnisse von Fragestellung eins und zwei eingegangen wird. 
Tabelle 1 zeigt modulweise die Selbsteinschätzung der Lehrpersonen bezüglich Einsatzhäufigkeit des Lernjournals und die ausgezählten Lernjournaleinträge der Schülerinnen und Schüler.

Tabelle 1: Modulweise Implementationshäufigkeit des Lernjournals (über den gesamten Interventionszeitraum)

\begin{tabular}{lccc}
\hline & \multicolumn{3}{c}{ Module } \\
\cline { 2 - 4 } & Leistung & Wagnis & Spiel \\
\hline Häufigkeitseinschätzung der Lehrperson & $8.3(4.7)$ & $8.6(3.7)$ & $5.0(0.7)$ \\
$\begin{array}{l}\text { Anzahl Lernjournaleinträge der Schülerinnen } \\
\text { und Schüler }\end{array}$ & $23.5(6.6)$ & $10.1(3.9)$ & $16.2(5.2)$ \\
\hline
\end{tabular}

Anmerkung: Mittelwerte mit Standardabweichungen in Klammern.

Die Schülerinnen und Schüler des Moduls Leistung nutzen das Lernjournal im Unterricht während dem Interventionszeitraum im Mittel 23.5 mal, also mehr als zweimal pro Woche. Die anderen beiden Module weisen im Mittel einen Eintrag pro Woche auf. Die selbsteingeschätzten Angaben der Lehrpersonen zum Sportheftgebrauch liegen unter den ausgezählten Lernjournaleinträgen. Bei diesen Angaben sind Verzerrungseffekte zu berücksichtigen und es bleibt unklar, auf welcher Zählweise die Selbsteinschätzungen der Lehrpersonen beruhen. Reflexionsphasen und Feedback werden im Unterricht laut Angaben der Lehrpersonen aller Module häufig eingesetzt (Reflexionsphasen: $M=3.19$, $S D=.66$; Feedback: $M=3.44, S D=.63$ ). Die Analyse der Implementationshäufigkeit zeigt, dass die Lehrpersonen das Lernjournal im Rahmen einer individuumsorientierten, reflexiven Sportvermittlung eingesetzt haben.

Personale Merkmale der Lehrperson und Implementationshäufigkeit

In Tabelle 2 sind die deskriptiven Resultate und jene der statistischen Tests der personalen Merkmale der beiden Lehrpersonengruppen «niedrige versus hohe Implementationshäufigkeit» dargestellt.

Unterschiede zwischen den beiden Gruppen zeigen sich einzig bezüglich der Berufserfahrung. Lehrpersonen mit mehr Berufserfahrung haben das Lernjournal im Rahmen einer individuumsorientierten, reflexiven Sportvermittlung im Unterricht häufiger eingesetzt als Lehrpersonen mit weniger Erfahrung. Obwohl die Lehrpersonen mit hoher Implementationshäufigkeit im Mittel älter sind als jene mit niedriger Implementationshäufigkeit, spielt das Alter der Lehrperson bezüglich der Implementationshäufigkeit eine untergeordnete Rolle. In allen drei Aspekten der Einstellung gegenüber der Intervention liegen die Angaben der Lehrpersonen mit hoher Implementationshäufigkeit im Mittel über jenen der Lehrpersonen mit niedriger Implementationshäufigkeit, ohne sich dabei signifikant zu unterscheiden. 
Tabelle 2: Ausprägungen und Unterschiede der Lehrpersonen in personalen Merkmalen in Abhängigkeit der Implementationshäufigkeit (IH)

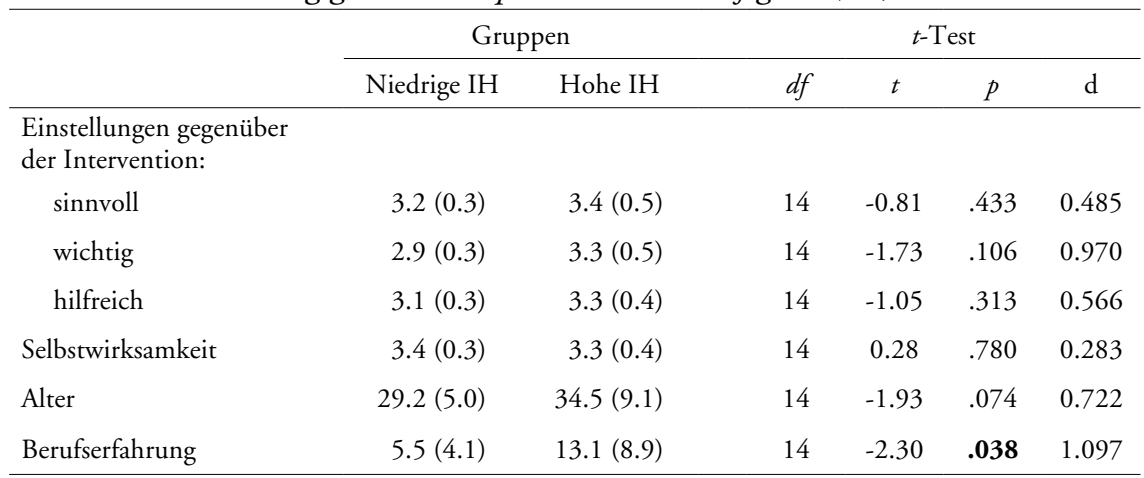

Anmerkungen: Mittelwerte mit Standardabweichungen in Klammern. $\mathrm{p}<.05$ sind fettgedruckt dargestellt.

Die fehlende Überzufälligkeit resultiert dabei eher aus der geringen Stichprobengrösse als aus zu geringen Effektstärken. Auch bezüglich der von den Lehrpersonen selbst wahrgenommenen Selbstwirksamkeit zeigen sich keine Unterschiede. Bezüglich personaler Merkmale der Lehrperson lässt sich somit festhalten, dass die Implementationshäufigkeit einer individuumsorientierten, reflexiven Sportvermittlung von der Berufserfahrung abhängt.

\section{Veränderung des sportbezogenen Fähigkeitsselbst- konzepts und des Körperselbstwerts in Abhängigkeit der Implementationshäufigkeit}

In Tabelle $3 \mathrm{a}$ und Tabelle $3 \mathrm{~b}$ sind die deskriptiven Resultate des sportbezogenen Fähigkeitsselbstkonzepts und des Körperselbstwerts in Abhängigkeit der Implementationshäufigkeit dargestellt (vgl. auch Abbildungen 2 und 3).

Tabelle 3a: Deskriptive Statistik des sportbezogenen Fähigkeitsselbstkonzepts in Abhängigkeit der Implementationshäufigkeit (IH)

\begin{tabular}{|c|c|c|c|c|c|c|}
\hline & \multicolumn{6}{|c|}{ Sportbezogenes Fähigkeitsselbstkonzept } \\
\hline & \multicolumn{3}{|c|}{ pre } & \multicolumn{3}{|c|}{ post } \\
\hline & $M(S D)$ & $95 \%-C I$ & $S E$ & $M(S D)$ & $95 \%-C I$ & $S E$ \\
\hline Niedrige IH & $3.08(0.60)$ & $2.93 / 3.23$ & 0.09 & $3.16(0.62)$ & $3.00 / 3.31$ & 0.09 \\
\hline Mittlere IH & $3.01(0.51)$ & $2.94 / 3.09$ & 0.04 & $3.05(0.56)$ & $2.98 / 3.13$ & 0.04 \\
\hline Hohe IH & $3.03(0.47)$ & $2.91 / 3.21$ & 0.07 & $3.06(0.46)$ & $2.91 / 3.22$ & 0.07 \\
\hline
\end{tabular}

Anmerkungen: Mittelwerte $(M)$ (mit Standardabweichungen $(S D)$ in Klammern), Unter- / Obergrenze des $95 \%$-Konfidenzintervalls $(95 \%$-CD) und Standardfehler $(S E)$. 
Tabelle 3b: Deskriptive Statistik des Körperselbstwerts in Abhängigkeit der Implementationshäufigkeit (IH)

\begin{tabular}{lccccccc}
\hline & \multicolumn{6}{c}{ Körperselbstwert } \\
\cline { 2 - 4 } \cline { 7 - 8 } & \multicolumn{3}{c}{ pre } & & \multicolumn{3}{c}{ post } \\
\cline { 2 - 3 } \cline { 7 - 8 } & $M(S D)$ & $95 \%-C I$ & $S E$ & & $M(S D)$ & $95 \%-C I$ & $S E$ \\
\hline Niedrige IH & $3.56(0.60)$ & $3.38 / 3.74$ & 0.09 & & $3.47(0.68)$ & $3.29 / 3.65$ & 0.10 \\
Mittlere IH & $3.41(0.65)$ & $3.22 / 3.50$ & 0.05 & & $3.47(0.62)$ & $3.38 / 3.56$ & 0.04 \\
Hohe IH & $3.31(0.72)$ & $3.14 / 3.50$ & 0.10 & & $3.43(0.68)$ & $3.25 / 3.61$ & 0.10 \\
\hline
\end{tabular}

Anmerkungen: Mittelwerte $(M)$ (mit Standardabweichungen $(S D)$ in Klammern), Unter- / Obergrenze des $95 \%$-Konfidenzintervalls $(95 \%-C l)$ und Standardfehler $(S E)$.

Tabelle 4: Ergebnisse der Varianzanalyse mit Messwiederholung bezüglich sportbezogenem Fähigkeitsselbstkonzept (SKSPO) und Körperselbstwert (KSWG)

\begin{tabular}{lcccccc}
\hline \multirow{2}{*}{ SKSPO } & \multicolumn{1}{c}{$S S$} & $d f$ & $M S$ & $F$ & $p$ & $\eta^{2}$ \\
\cline { 2 - 6 } Zeit & & & & & & \\
Gruppe & 0.17 & 1,291 & 0.17 & 2.67 & .050 & .009 \\
Zeit x Gruppe & 0.6 & 2,291 & 0.3 & 0.59 & .280 & .004 \\
& 0.06 & 2,291 & 0.03 & 0.43 & .324 & .003 \\
KSWG & & & & & & \\
\hline Zeit & & & & & & \\
Gruppe & 0.05 & 1,294 & 0.05 & 0.47 & .250 & .002 \\
Zeit x Gruppe & 0.97 & 2,294 & 0.48 & 0.66 & .259 & .004 \\
\hline
\end{tabular}

Anmerkungen: Die ausgewiesenen $p$-Werte beziehen sich auf einseitige Signifikanztests für gerichtete Fragestellungen. $p<.05$ sind fettgedruckt dargestellt.

Bezüglich der Veränderung des sportbezogenen Fähigkeitsselbstkonzepts unterscheiden sich die Implementationsgruppen nicht (kein signifikanter Interaktionseffekt, vgl. Tabelle 4). Die Hypothese wird abgelehnt. Eine hohe Implementationshäufigkeit einer individuumsorientierten, reflexiven Sportvermittlung führt beim sportbezogenen Fähigkeitsselbstkonzept im Vergleich zu einer mittleren oder niedrigen Implementationshäufigkeit nicht zu einer grösseren Veränderung. 


\section{Sportbezogenes Fähigkeitsselbstkonzept}

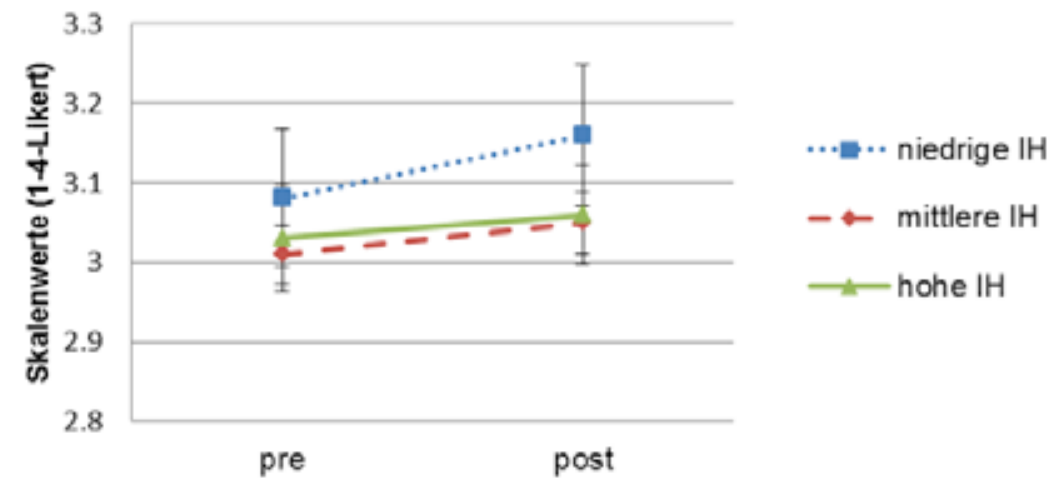

Abbildung 2: Mittelwerte des sportbezogenen Fähigkeitsselbstkonzepts in Abhängigkeit der Implementationshäufigkeit (IH). Die Fehlerbalken zeigen den Standardfehler des Mittelwerts.

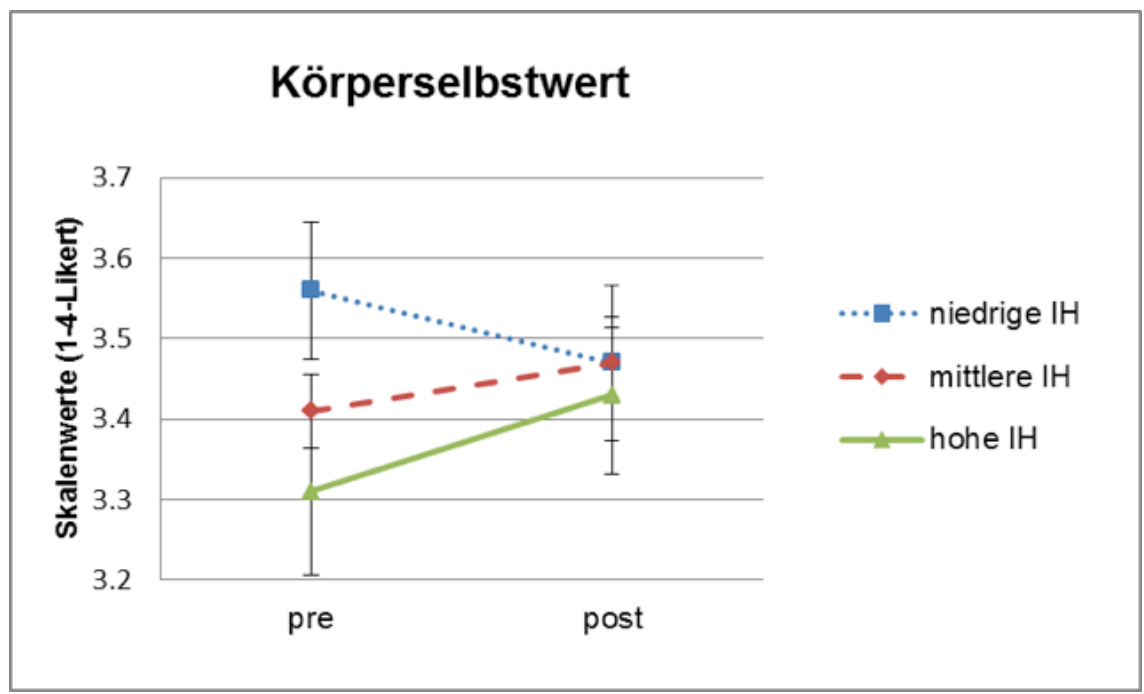

Abbildung 3: Mittelwerte des Körperselbstwerts in Abhängigkeit der Implementationshäufigkeit (IH). Die Feblerbalken zeigen den Standardfehler des Mittelwerts. 
Im Gegensatz dazu zeigen sich bezüglich der Veränderung des Körperselbstwerts bedeutsame Unterschiede zwischen den drei Implementationsgruppen (vgl. Tabelle 4: signifikanter Interaktionseffekt bei statistisch nicht unterschiedlichen Baseline-Werten $(F(2,300)=.80, p=.151))$. Während der Körperselbstwert der Schülerinnen und Schüler mit hoher Implementationshäufigkeit deutlich und dieser von Schülerinnen und Schülern mit mittlerer Implementationshäufigkeit leicht zunimmt, nimmt der Körperselbstwert von Schülerinnen und Schülern mit niedriger Implementationshäufigkeit ab (vgl. Abbildung 3). Die Hypothese wird angenommen. Eine hohe Implementationshäufigkeit einer individuumsorientierten, reflexiven Sportvermittlung geht im Vergleich zu einer mittleren oder niedrigen Implementationshäufigkeit mit grösseren Veränderungen im Körperselbstwert einher.

Allerdings ist zu beachten, dass sich gruppenunabhängig über die Zeit sowohl bezüglich des sportbezogenen Fähigkeitsselbstkonzepts als auch bezüglich des Körperselbstwerts keine Verbesserungen ausweisen lassen (jeweils kein signifikanter Haupteffekt der Zeit, vgl. Tabelle 4). Die Intervention hat unabhängig von den verschiedenen Implementationshäufigkeiten nicht zu überzufälligen Veränderungen im Selbstkonzept geführt.

Abschliessend ist festzuhalten, dass sich eine häufige individuumsorientierte, reflexive Sportvermittlung der Lehrperson im Gegensatz zu einer weniger häufigen individuumsorientierten, reflexiven Sportvermittlung positiv auf den Körperselbstwert, jedoch nicht auf das sportbezogene Fähigkeitsselbstkonzept von Schülerinnen und Schülern auswirkt.

\section{Diskussion}

Die vorliegende Studie befasste sich mit der Messung und Erklärung von Implementation im Rahmen einer Intervention zu Selbstkonzept förderndem Sportunterricht. Die vorausgehende Analyse zeigt, dass Lehrpersonen eine individuumsorientierte, reflexive Sportvermittlung anhand des Lernjournals im Unterricht einsetzen. Die inferenzstatistischen Ergebnisse weisen erstens daraufhin, dass eine hohe Implementationshäufigkeit mit einer grossen Berufserfahrung der Lehrperson einhergeht. Eine individuell-reflexive Förderung im Sportunterricht scheint erst wahrscheinlich, wenn eine gewisse (Sport-)Unterrichtserfahrung vorhanden ist. Dies widerspricht der von Lütgert und Stephan (1983, S. 508) beschriebenen Tendenz von Lehrpersonen, routiniertes Verhalten bei curricularen Innovationen beizubehalten, stimmt jedoch mit den Aussagen von Bauer, Kopka und Brindt (1996) überein, nach welchen Handlungsrepertoire und Einstellungen zur Wissenschaft mit der Berufserfahrung von Lehrpersonen eng verknüpft sind. Diese Erkenntnis ist bei der Planung und Gestaltung zukünftiger Interventionsstudien zu berücksichtigen und Lehrpersonen mit weniger Berufserfahrung sind entsprechend zu instruieren. 
Die Ergebnisse zeigen zweitens, dass sich eine häufige individuumsorientierte, reflexive Sportvermittlung der Lehrperson im Gegensatz zu einer weniger häufigen individuumsorientierten, reflexiven Sportvermittlung positiv auf den Körperselbstwert von Schülerinnen und Schülern auswirkt. Dieses Ergebnis entspricht bisherigen Erkenntnissen bezüglich des Zusammenhangs zwischen Implementationshäufigkeit und selbstkonzeptnahen Kompetenzen (z. B. Drössler et al., 2007) und verdeutlicht die Relevanz einer hohen Implementationshäufigkeit und detaillierter Implementationskenntnisse zur Gestaltung und Erklärung von Interventionsstudien. Beim sportbezogenen Fähigkeitsselbstkonzept finden sich in Abhängigkeit der Implementationshäufigkeit keine Veränderungen. Dieses Ergebnis ist einerseits auf die geringe gruppenübergreifende Veränderung des sportbezogenen Fähigkeitsselbstkonzepts zurückzuführen. Andererseits entspricht das sportbezogene Fähigkeitsselbstkonzept, im Gegensatz zum evaluativen Körperselbstwert, einer deskriptiven Komponente des Selbstkonzepts und ist somit stärker abhängig von einem äusseren Kriterium, hier der realen sportlichen Leistungsfähigkeit. Die sportliche Leistungsfähigkeit kann jedoch insbesondere bei koordinativen und taktischen Fähigkeiten innerhalb des kurzen Interventionszeitraums höchstens marginal verbessert werden, weshalb der Interventionszeitraum wohl auch für Veränderungen des sportbezogenen Fähigkeitsselbstkonzepts zu kurz war (bez. Veränderungen des Fähigkeitsselbstkonzepts vgl. Filipp, 2006).

Sowohl beim Körperselbstwert als auch beim sportbezogenen Fähigkeitsselbstkonzept finden sich unabhängig von den Implementationsgruppen keine übergreifenden Veränderungen und damit kein grundsätzlicher Effekt einer individuumsorientierten, reflexiven Sportvermittlung auf die hier untersuchten Aspekte des Selbstkonzepts (vgl. Conzelmann et al., 2011). Allerdings ist zu bedenken, dass das Erbringen von Nachweisen bei Interventionsstudien im schulischen Setting Schwierigkeiten aufweist. In diesem Sinne weisen die hier unter einer differenziellen Implementationsperspektive gewonnenen Erkenntnisse trotz allem darauf hin, dass eine häufige individuumsorientierte, reflexive Sportvermittlung bei gewissen, in erster Linie evaluativen Aspekten des Selbstkonzepts durchaus Veränderungen zu erzeugen vermag. Die vorliegende Studie zeigt zudem auf, dass die Berücksichtigung der Implementation bei der Erklärung von Ergebnissen von Interventionsstudien im (Sport-)Unterricht entscheidend ist. Mit diesen Erkenntnissen leistet die vorliegende Studie einen Beitrag zur Implementationsforschung innerhalb der Unterrichtswissenschaft und zur Selbstkonzeptentwicklung im Sportunterricht.

Kritisch zu betrachten sind in der vorliegenden Studie insbesondere der geringe Stichprobenumfang der Lehrpersonen und der kurze Interventionszeitraum von zehn Wochen, welche den Nachweis von Programmen erschweren. Die Implementationshäufigkeit hätte zudem durch weitere objektive Kriterien erhoben (z. B. Analyse von uns nur begrenzt vorliegenden Unterrichtsprotokollen der Lehrperson) oder im Rahmen einer umfassenden, qualitativen Analyse 
der gesamten Implementationsgenauigkeit durchgeführt werden können. Dies hätte eine tiefergehende Analyse der Ergebnisse ermöglicht, was jedoch aufgrund der Datenlage und -qualität nicht möglich war. Wünschenswert sind zukünftige Interventionsstudien, die diese Aspekte berücksichtigen und damit sowohl die Implementationsforschung als auch das Wissen um die Förderung des Selbstkonzepts im Sportunterricht weiter vertiefen.

\section{Anmerkungen}

1 Das sechste und neunte Item werden aufgrund sehr schlechter Interitemkorrelationen mit den anderen sieben Items (Item 6: $r_{1}=.13, r_{2}=-.06, r_{3}=.00, r_{4}=-.08, r_{5}=.14, r_{7}=-.28$, $r_{8}=.22, r_{9}=.22$; Item 9: $r_{1}=.11, r_{2}=-.37, r_{3}=.09, r_{4}=.26, r_{5}=-.02, r_{6}=.22, r_{7}=.30$, $\left.r_{8}=.16\right)$ für die weitere Skalenbildung ausgeschlossen.

2 Die Lernjournale wurden für jedes Modul unterschiedlich gestaltet und ermöglichen so eine unterschiedliche Anzahl Maximaleinträge. Zur Auswertung beider Fragestellungen erfolgte die Gruppeneinteilung deshalb unter Berücksichtigung der jeweiligen Module.

3 Da die Module Wagnis und Spiel eine ungerade Anzahl Probanden aufweisen, wurde beim Mediansplit der jeweilige Medianwert der Gruppe mit dem nächst entfernten Wert zugeteilt. Im Modul Leistung bestand in der Rangordnung zwischen dem zweiten und dritten von sechs Werten eine grosse Distanz, weshalb hier der dritte Wert (welcher eigentlich unter dem Median liegt) aus inhaltlichen Gründen trotzdem der Gruppe über dem Median zugeteilt wurde.

4 Bivariate Korrelationen zwischen der objektiven Anzahl Lernjournaleinträge (AS) und den Selbsteinschätzungen der Lehrpersonen betreffend Einsatzhäufigkeit des Lernjournals $(\mathrm{S})$ sowie zwischen $S$ und Reflexion (R) und Feedback (F). Zur Berechnung der ersteren wurden die Angaben der Lehrperson ihren Schülerinnen und Schülern zugeteilt: $\left({ }^{*} p<.05\right)$ Modul Leistung: $r_{A S, S}=.19^{*}, r_{S, R}=.53, r_{S, F}=.24, r_{R F}=.11$

Modul Wagnis: $r_{A S, S}=.02, r_{S, R}=.50, r_{S, F}=.50, r_{R F}=.50$

Modul Spiel: $r_{A S, S}=.70^{*}, r_{S, R}=.60, r_{S, F}=.60, r_{R F}=.17$

\section{Literatur}

Aust, K., Watermann, R. \& Grube, D. (2010). Selbstkonzeptentwicklung und der Einfluss von Zielorientierungen nach dem Übergang in die weiterführende Schule. Zeitschrift für Pädagogische Psychologie, 24, 95-109.

Bandura, A. (1997). Self-efficacy: The exercise of control. New York: Freeman.

Bauer, K.-O., Kopka, A. \& Brindt, S. (1996). Pädagogische Professionalität und Lehrerarbeit: Eine qualitativ empirische Studie über professionelles Handeln und Bewusstsein. Weinheim: Juventa.

Böttcher, W. (2002). Kann eine ökonomische Schule auch eine pädagogische sein? Schulentwicklung zwischen Neuer Steuerung, Organisation, Leistungsevaluation und Bildung. Weinheim: Juventa.

Brettschneider, W.-D. \& Gerlach, E. (2004). Sportliches Engagement und Entwicklung im Kindesalter. Eine Evaluation zum Paderborner Talentmodell. Aachen: Meyer \& Meyer.

Brohm, M. (2006). Evaluation von Schüler- und Lehrertrainingsprogrammen: Bedingungen, theoretische Ansätze, Forschungsdesigns. In W. Böttcher, H. G. Holtappels \& M. Brohm (Hrsg.), Evaluation im Bildungswesen: Eine Einfuihrung in Grundlagen und Praxisbeispiele (S. 245-263). Weinheim: Juventa.

Bucher, W. (1997). Lehrmittelreihe Sporterziehung. Bern: EDMZ.

Conzelmann, A., Schmidt, M. \& Valkanover, S. (2011). Persönlichkeitsentwicklung durch Schulsport. Theorie, Empirie und Praxisbausteine der Berner Interventionsstudie Schulsport (BISS). Bern: Huber. 
Dane, A. V. \& Schneider, B. H. (1998). Program integrity in primary and early secondary prevention: Are implementation effects out of control? Clinical Psychology Review, 18, 23-45.

Deutscher Sportbund (Hrsg.). (2006). DSB-SPRINT-Studie. Eine Untersuchung zur Situation des Schulsports in Deutschland. Aachen: Meyer \& Meyer.

Döring, K.W. (1989). Lehrerverhalten. Ein Lehr-und Arbeitsbuch (9. Aufl.). Weinheim: Deutscher Studien Verlag.

Drössler, S., Jerusalem, M. \& Mittag, W. (2007). Förderung sozialer Kompetenzen im Unterricht: Implementation eines Lehrerfortbildungsprojekts. Zeitschrift für Pädagogische Psychologie, 21, 157-168.

Erziehungsdirektion Kanton Bern. (2004). Lehrplan für die Volksschule des Kantons Bern 1995 Zugriffam 12.01.2012 unterhttp://www.erz.be.ch/erz/de/index/kindergarten_volksschule/ kindergarten_volksschule/lehrplaene/volksschule.assetref/content/dam/documentsERZ/ AKVB/de/03_Lehrplaene_Lehrmittel/lehrplaene_lehrmittel_vs_sport_d.pdf

Euler, D. \& Sloane, P. F. E. (1998). Implementation als Problem der Modellversuchsforschung. Unterrichtswissenschaft, 26, 312-326.

Filipp, S.-H. (1979). Entwurf eines heuristischen Bezugsrahmens für Selbstkonzeptfoschung: Menschliche Informationsverarbeitung und naive Handlungstheorie. In S.-H. Filipp (Hrsg.), Selbstkonzept-Forschung (S. 129-153). Stuttgart: Klett-Cotta.

Filipp, S.-H. (2006). Entwicklung von Fähigkeitsselbstkonzepten. Zeitschrift für Pädagogische Psychologie, 20, 65-72.

Fox, K. R. (1990). The physical self-perception profile manual. DeKalb: Office for Health Promotion, Northern Illinois University.

Fox, K. R. (2000). The effects of exercise on physical self-perceptions and self-esteem. In S. J. H. Biddle, K. R. Fox \& S. H. Boutcher (Eds.), Physical activity and psychological well-being (pp. 88-117). London: Routledge \& Kegan Paul.

Gollwitzer, M. \& Jäger, R. S. (2007). Evaluation: Workbook. Weinheim: Beltz.

Gräsel, C. \& Parchmann, I. (2004). Implementationsforschung - oder: der steinige Weg, Unterricht zu verändern. Unterrichtswissenschaft: Zeitschrift für Lernforschung, 32, 196-214.

Gräsel, C., Parchmann, I., Puhl, T., Baer, A., Fey, A. \& Demuth, R. (2004). Lehrerfortbildungen und ihre Wirkungen auf die Zusammenarbeit von Lehrkräften und die Unterrichtsqualität. In J. Doll \& M. Prenzel (Hrsg.), Bildungsqualität von Schule. Lehrerprofessionalisierung, Unterrichtsentwicklung und Schülerförderung als Strategien der Qualitätsverbesserung (S. 133-151). Münster: Waxmann.

Harter, S. (1985). Manual for the self-perception profile for children. Denver, CO: University of Denver.

Herzog, W. (2002). Zeitgemässe Erziehung - Die Konstruktion pädagogischer Wirklichkeit. Weilerswist: Velbrück Wissenschaft.

Lüdtke, O. \& Köller, O. (2002). Individuelle Bezugsnormorientierung und soziale Vergleiche im Mathematikunterricht: Einfluss unterschiedlicher Referenzrahmen auf das fachspezifische Selbstkonzept der Begabung. Zeitschrift für Entwicklungspsychologie und Pädagogische Psychologie, 34, 156-166.

Lütgert, W. \& Stephan, H.-U. (1983). Implementation und Evaluation von Curricula: deutschsprachiger Raum. In U. Hameyer, K. Frey \& H. Henning (Hrsg.), Handbuch der Curriculumforschung. Erste Ausgabe. Übersichten zur Forschung 1970-1981 (S. 501-520). Weinheim: Beltz.

Mihalic, S. F. (2004). The importance of implementation fidelity. Emotional \& Behavioral Disorders in Youth, 4, 83-90.

Paul, G. \& Volk, T. L. (2002). Ten years of teacher workshops in a environmental problemsolving model: Teacher implementations and perceptions. The Journal of Environmental Education, 33, 10-20. 
Radtke, F. O. (1996). Wissen und Können. Die Rolle der Erziehungswissenschaft in der Erziehung. Opladen: Leske + Budrich.

Rheinberg, F. (2008). Bezugsnormen und die Beurteilung von Lernleistung. In W. Schneider \& M. Hasselhorn (Hrsg.), Handbuch der pädagogischen Psychologie (S. 178-186). Göttingen: Hogrefe.

Reinmann-Rothmeier, G. \& Mandl, H. (1998). Wenn kreative Ansätze versanden: Implementation als verkannte Aufgabe. Unterrichtswissenschaft, 26, 292-311.

Schmitz, G. S. \& Schwarzer, R. (2002). Individuelle und kollektive Selbstwirksamkeitserwartung von Lehrern. In M. Jerusalem \& D. Hopf (Hrsg.), Selbstwirksamkeit und Motivationsprozesse in Bildungsinstitutionen [Themenheft]. Zeitschrift für Pädagogik, 44 (S. 192-214). Weinheim: Beltz.

Shavelson, R. J., Hubner, J. J. \& Stanton, G. C. (1976). Self-concept: Validation of construct interpretations. Review of Educational Research, 46, 407-441.

Sonstroem, R. J. \& Morgan, W. P. (1989). Exercise and self-esteem: Rationale and model. Medicine and Science in Sports and Exercise, 21 (3), 329-337.

Stiller, J. \& Alfermann, D. (2005). Selbstkonzept im Sport. Zeitschrift für Sportpsychologie, $12,119-126$.

Stiller, J. \& Alfermann, D. (2008). Inhalte und Struktur des physischen Selbstkonzepts. In A. Conzelmann \& F. Hänsel (Hrsg.), Sport und Selbstkonzept. Struktur, Dynamik und Entwicklung (S. 14-25). Schorndorf: Hofmann.

Zedler, P., Fischler, H., Kirchner, S. \& Schröder, H.-J. (2004). Fachdidaktisches Coaching - Veränderungen von Lehrerkognitionen und unterrichtlichen Handlungsmustern. In J. Doll \& M. Prenzel (Hrsg.), Bildungsqualität von Schule. Lehrerprofessionalisierung, Unterrichtsentwicklung und Schülerförderung als Strategien der Qualitätsverbesserung (S. 114-132). Münster: Waxmann.

Schlagworte: Implementation, Selbstkonzept, Sportunterricht

\section{L'implémentation d'une intervention pour changer le concept de soi en éducation physique}

\section{Ré sumé}

Les études portant sur le développement du concept de soi rapportent rarement les conditions d'implémentation des interventions visant à stimuler ce développement. La présente étude pallie ce manque en examinant les caractéristiques et la fréquence avec laquelle une intervention promouvant le concept de soi en éducation physique est implémentée $\left(\mathrm{N}_{\text {Enseignant }}=16, \mathrm{~N}_{\text {Etudiant }}=309\right)$ tout en mesurant les effets produits. Les résultats montrent que les enseignants d'éducation physique mettent en place un enseignement réflexif et orienté vers l'individu. La fréquence d'interventions des enseignants dépend de leurs expériences professionnelles. L'augmentation de la fréquence d'interventions implémentées va de pair avec un renforcement de l'estime de soi des élèves dans le domaine corporel sans modifier le concept de soi lié à leurs compétences sportives.

Mots-clés: Conditions d'implémentation, concept de soi (self-concept), éducation physique 


\title{
L'implementazione di un intervento per modificare il concetto di sé nell'educazione fisica
}

\section{Riassunto}

Nell'ambito degli studi sullo sviluppo del concetto di sé mancano spesso indicazioni relative all'effettiva messa in pratica degli interventi; lo studio presentato in questo articolo si concentra proprio su questa mancanza di controllo nell'implementazione. Questa ricerca si occupa della misurazione e della spiegazione della frequenza di attuazione di interventi che portano a un miglioramento del concetto di sé in educazione fisica $\left(N_{\text {maestri }}=16, N_{\text {studenti }}=309\right)$. I risultati indicano che i maestri attuano un approccio alleducazione fisica riflessivo e orientato all'individuo. La frequenza di attuazione di tale approccio dipende dalla loro esperienza professionale. Una maggiore frequenza di attuazione porta ad un maggior cambiamento dell'autostima degli studenti rispetto al proprio corpo, ma non ad un cambiamento del concetto di sé in relazione alla loro abilità sportiva.

Parole chiave: Implementazione, concetto di sé (self-concept), educazione fisica

\section{Implementation of an intervention towards altering the self- concept in physical education}

\begin{abstract}
Studies of interventions fostering self-concept development often omit to report information on implementation conditions. The present study contributes to overcome this deficit, by investigating the implementation frequency and the effects of an interventionaimed at improving childrens' self-concept within a physical education $\left(\mathrm{N}_{\text {Teachers }}=16, \mathrm{~N}_{\text {Students }}=309\right)$. Results reveal that physical education is being taught in an individual-oriented and reflexive way. The frequency of the implementation depends on the teacher's professional experience. Frequent intervention implementation is linked with significant change in students' self-concept, but not with perceived physical ability.
\end{abstract}

Key words: Implementation, self-concept, physical education 
